\title{
Update in the myeloproliferative neoplasms
}

\author{
Authors: Claire N Harrison ${ }^{A}$ and Mary F McMullin ${ }^{B}$
}

The differential diagnosis of haematological abnormalities, such as leucocytosis, erythocytosis, thrombocytosis or indeed anaemia, is wide and disarming. Here we report on significant updates in the differential diagnosis of erythrocyosis and thrombocytosis presenting a simplified schema for the clinician. We then move to discuss significant advances in this field which have followed a series of key molecular findings, most specifically those affecting the JAK/STAT pathway.

KEYWORDS: Polycythaemia, erythrocytosis, thrombocytosis, myelofibrosis, myeloproliferative

\section{Introduction}

Myeloproliferative neoplasms (MPNs) are clonal haematological diseases characterised by the overproduction of mature blood cells and a chronic course. They were first recognised as a distinct group of clinical entities by William Dameshek in 1951. At this time chronic myeloid leukaemia (CML) was also included in their number but, with the familiar description of the Philadelphia chromosome in CML, the other conditions were then re-named Philadelphia-negative myeloproliferative disorders. The World Health Organization has recently modified the nomenclature from myeloproliferative disorder to myeloproliferative neoplasm (MPN), reflecting their malignant nature. MPNs include polycythaemia vera (PV), primary myelofibrosis (PMF) and essential thrombocythaemia (ET), and the rarer entities, chronic neutrophilic leukaemia, chronic eosinophilic leukaemia and chronic myeloproliferative neoplasm, unclassifiable as well as mast cell diseases (Box 1). Here, we consider the more common so-called classical Philadelphia-negative MPNs: ET, PV and PMF. These can broadly be identified by the proliferation of a single cell type with disease-defining phenotype: erythrocytes in PV, platelets in ET and fibroblasts in PMF.

\section{Advances in molecular biology}

An acquired point mutation in the JAK2 (JAK2V617F) gene was described in 2005 and occurs in most patients with PV

Authors: A professor of haematology, Department of Haematology Guy's and St Thomas' Hospital, London, UK; B professor of haematology, CCRCB, Queen's University, Belfast, UK and almost half of those with ET or PMF. ${ }^{1}$ This is a somatic mutation that occurs in a part of the protein molecule, the pseudokinase domain, which is normally folded upon itself and inhibits the active kinase site. In the process of normal intracellular signal transduction, the kinase and pseudokinase domains are postulated to become distanced from each other, resulting in kinase activity and thence signal relay. The mutation, which is found within what is thought to be the inhibitory pseudokinase site, leads to a protein with intrinsic kinase activity enhancing cytokine-mediated pathways such as JAK/STAT, PI3K/AKT/mTOR and MAPK/ERK, affecting cell division, apoptosis and protein synthesis. Deregulated JAK2 also directly phosphorylates histone H3Y41 in the nucleus, causing displacement of HP1 $\alpha$ and leading to additional oncogenic effects. ${ }^{2}$

The majority of the small number of patients with PV who lack the JAK2V617F mutation have one of a number of mutations found in exon 14 of this same molecule. ${ }^{3}$ Other somatic mutations have since been found, such as in exon 10 of the thrombopoietin receptor MPL in about 5-10\% of cases of ET and PMF. ${ }^{4}$ A series of highly prevalent mutations in Calreticulin $(C A L R)$ have very recently been described which appear to be present in the majority of patients lacking the JAK2V617F mutation. ${ }^{5,6}$ CALR represents a novel therapeutic target of great interest in the field. The mutations which have been described to date, of which there are many, affect the calcium binding domain at the C-terminus of the molecule. In cell lines, CALR mutations are associated with enhanced JAK/ STAT activation and in mouse models, recapitulate disease phenotype. There is also increasing evidence of additional mutations occurring in MPN which affect epigenetic regulation and the spliceosome in the MPNs, which may be of prognostic significance. ${ }^{7}$ Such mutations, unlike all those mentioned previously, lack the relative diagnostic specificity for MPN over

\section{Box 1. Myeloproliferative neoplasms.}

Polycythaemia vera

Chronic idiopathic myelofibrosis

Essential thrombocythaemia

Chronic neutrophilic leukaemia

Chronic eosinophilic leukaemia

Mast cell disorders

Myeloproliferative neoplasm, unclassifiable 


\section{Box 2. Causes of an erythrocytosis.}

\section{Causes of absolute erythrocytosis (ie red cell mass $125 \%$ of predicted)}

\section{Primary (abnormality within RBCs)}

Congenital

Truncated erythropoietin receptor

Mutations within LNK

Acquired

Polycythaemia vera

\section{Secondary (abnormality outside RBCs)}

Congenital

Abnormal haemoglobin with increased oxygen affinity

Reduced 2,3-bisphosphoglycerate

Mutation in von Hippel-Lindau gene

Mutations in proline dehydroxylase genes

Mutations in hypoxia inducible factor genes

Acquired (increased erythropoietin)

Conditions causing low oxygen levels - high altitude, chronic lung disease, some congenital heart diseases Renal disease - tumours (hypernephroma), cysts (usually benign), hydronephrosis, following kidney transplantation Liver disease - hepatoma, cirrhosis, hepatitis

Tumours - bronchial cancer, fibroids in the uterus, cerebellar haemangiomata

Endocrine abnormalities - Cushing's syndrome, phaeochromocytoma

Drugs - erythropoietin and androgens

Idiopathic (undefined primary or secondary) May resolve or pathology may be masked initially

\section{Causes of apparent erythrocytosis (ie normal red cell mass} but elevated haematocrit)

Normal variant

Early absolute erythrocytosis

Obesity, fluid loss, diuretics, smoking, hypertension, alcohol, renal disease, psychological stress

$\mathrm{RBCS}=$ red blood cells.

other myeloid conditions. For example, JAK2V617F is identified in only rare subgroups of patients with myelodysplasia but mutations in genes involved in epigenetic pathways such as $S R S F 2, E Z H 2$, and ASXL1 are frequently observed in other myeloid malignancies.

The malignant phenotype seen in MPN therefore results from a combination of genetic abnormalities and epigenetic modifications leading to a dysregulation of critical genes contributing to proliferation and cell death. These recent findings paved the way for faster and more accurate diagnostics and are beginning to influence both therapeutics and prognosis.

\section{Making a diagnosis of a myeloproliferative neoplasm}

Suspecting a diagnosis is very important for these conditions, which may present in protean manners from asymptomatic persistently abnormal blood counts, the presence of splenomegaly, to atypical thrombotic events such as splanchnic vein thrombosis where blood counts may in fact be normal. The latter is a scenario where it is particularly important to consider an MPN since in the absence of a clear underlying cause they are the most common underlying factor. Screening for MPN does not however form part of a standard thrombophilia screen as studies have shown this to be inappropriate. A diagnostic algorithm for MPN is shown in Fig 1; here a heavy reliance is placed upon molecular tests and bone marrow histomorphology. Exclusion of reactive causes is also important especially in the increasingly smaller numbers of patients lacking a specific molecular marker and where interpretation of the marrow trephine biopsy may lack specificity. It can sometimes be difficult to differentiate between ET and masked PV, for example when factors such as renal impairment are present, which will reduce erythropoiesis, careful evaluation of patients is important to make sure this is not missed.

\section{Management of essential thrombocythaemia and polycythaemia vera}

This patient group benefits from aggressive risk management for vascular disease. Low-dose aspirin is widely used for both of these groups of patients but while there is evidence from the ECLAP study for this strategy in $\mathrm{PV},{ }^{8}$ this remains controversial in ET. ${ }^{9}$ Excess bleeding is a risk, particularly for patients with very high platelet counts, and also occurs with more modestly elevated platelet counts. The target haematocrit for venesection in PV has been controversial, but the recently reported CYTO PV study strongly supports control of the haematocrit to at least $0.45 .^{10}$

Cytoreductive therapy in ET and PV is used to reduce the risk of thrombosis, control disease-related symptoms and where possible reduce the risk of progression. Since thrombosis is the most commonly encountered complication, it is the most rational endpoint on which to base risk stratification, and a number of such risk stratification schemes have been proposed for this purpose. Any novel markers for thrombosis risk or survival should be robust and easily measurable; current candidates include the leucocyte count, ${ }^{11}$ reticulin grade, ${ }^{12,13}$ and JAK2V617F allele burden, ${ }^{13}$ and for ET the presence of the $C A L R$ seem to correlate with better prognosis and lower risk of thrombosis. ${ }^{5}$ The impact of conventional risk factors for atherosclerosis have been assessed in MPN with variable results and whether these risk factors should contribute to risk group allocation for ET and PV patients - especially if they are wellcontrolled - is unclear.

Choice of cytoreductive therapy is relatively limited and a better evidence base is definitely desirable. Interferon alpha (IFN) and hydroxycarbamide or hydroxyurea (HU) form the mainstay of therapy. Studies of pegylated IFN alpha2a (Pegasys) in PV and ET show high levels of complete haematologic response rate and good tolerance of the drug, particularly when started at low doses. ${ }^{14,15}$ Of note, there appears to be preferential targeting of the JAK2V617F mutated clone in this context. ${ }^{16}$ This suggests that Pegasys may be effective when other therapies have failed or not been tolerated and that it may in the future as more targeted therapies perhaps challenge the position of $\mathrm{HU}$ as first line therapy. However this will require large randomised trials with comprehensive evaluation for long term side effects. Several such studies are underway. 

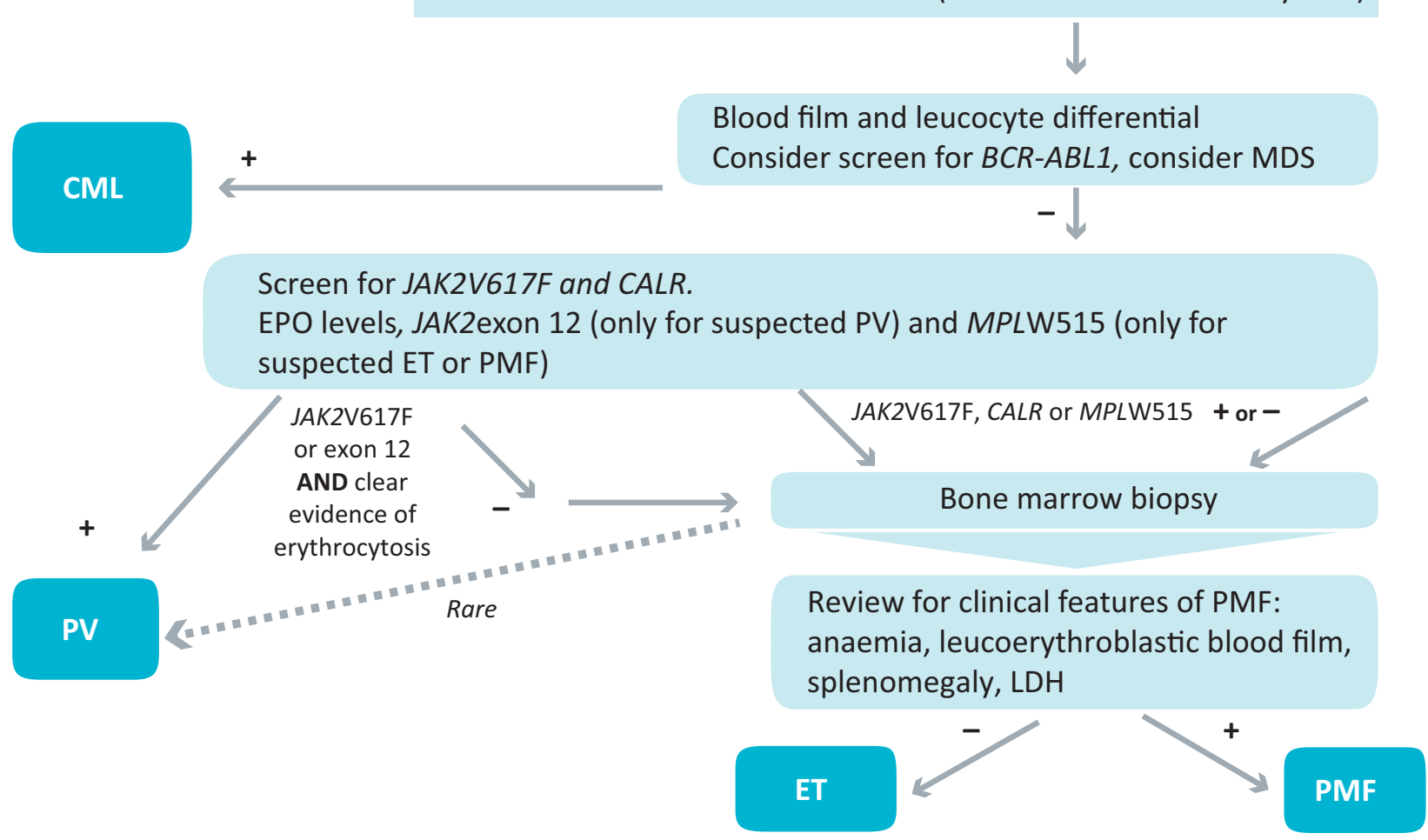

Fig 1. Algorithm for diagnosis of MPN. CML = chronic myeloid leukaemia; $E T=$ essential thrombocythaemia; $L D H=$ lactate dehydrogenase; $M N P=$ myeloproliferative neoplasm; PMF = primary myelofibrosis; $\mathrm{PV}=$ polycythaemia vera.

A significant number of JAK inhibitors are now at varying stages of clinical evaluation with most data available for patients with myelofibrosis (see below). There are data supporting their ability to control myeloproliferation in patients with PV and ET to prevent thrombosis, affect the probability of accelerated/more aggressive disease, such as post-ET or post-PV myelofibrosis (MF), or indeed leukaemia. In an initial study with the JAK1 and JAK2 inhibitor, ruxolitinib, significant rates of reduction of splenomegaly and symptom scores were achieved but here all patients had leucocyte counts below $10 \times 10^{9} / 1$ and $41 \%$ achieved a complete response with platelets less than $400 \times 10^{9} / 1$, and no thrombotic events are reported thus far. ${ }^{17}$ Two large phase-III commercially sponsored studies of ruxolitinib for patients with PV are fully recruited and the results are awaited. There is one on-going academic study of ruxolitinib in ET patients who are resistant or refractory to $\mathrm{HU}$.

The histone deacetylase (HDAC) inhibitors such as vorinostat ${ }^{18}$ and givinostat ${ }^{19}$ have been used in patients, though toxicities with these agents are variable and with vorinostat lead to high discontinuation rates. The oral telomerase inhibitor imetelstat was investigated in ET, demonstrating molecular responses but also significant rates of haematological toxicity. ${ }^{20}$ Further studies of sufficient duration to permit the evaluation of the safety and efficacy of these and other novel agents are certainly needed; however, these must incorporate health economic assessments, long term safety and biologically meaningful endpoints beyond the control of blood counts to be credible.

\section{Management of myelofibrosis}

Until recently, therapeutic options for patients with MF consisted of allogeneic stem cell transplantation, cytoreductive agents, splenectomy or splenic irradiation, and management of anaemia with transfusions, erythropoiesis-stimulating agents, androgens and immunomodulatory agents. ${ }^{21}$ Several prognostic scoring systems exist to support the management of MF. A likely modification to these scores will incorporate screening for mutations in the genes ASXL1, EZH2, IDH1/2, and SRSF2; in a recent study these were associated with worse survival outcomes with a greater likelihood of transformation to acute leukaemia. ${ }^{7}$ However at present screening for such mutations is not carried out in routine practice nor is it incorporated into prognostic scores. CALR mutation status is also going to have an impact here as its presence appears to be associated with a better prognosis in MF.

Large phase III trials have never previously been conducted in MF but the discovery of the role JAK/STAT activation in its pathogenesis led to development and approval of the first small-molecule JAK inhibitors following phase III trials, known as the Controlled Myelofibrosis Study with Oral JAK Inhibitor 
Treatment (COMFORT) trials. ${ }^{22,23}$ Intriguingly, clinical responses in both studies were observed regardless of JAK2 V617F mutation status. The COMFORT trials also demonstrated that, in addition to the profound effects on splenomegaly, the JAK 1 and 2 inhibitor ruxolitinib provided statistically significant and very rapid improvements in patients' symptoms and quality of life and there is increasingly strong evidence for a survival benefit associated with this therapy. Unlike BCR/ABL kinase inhibition there is, as yet, no evidence that JAK inhibition alone is curative for MPN patients. Several other such agents are currently in various stages of development. ${ }^{24}$

Three important safety concerns have recently arisen with regard to JAK inhibition: the existence of a 'withdrawal syndrome', neurological toxicity and lastly the risk of infections. Early reports suggested an apparent risk of a severe inflammatory syndrome with poor outcome after ruxolitinib withdrawal. ${ }^{25}$ COMFORT-I trial patients recorded symptom scores on a daily basis and demonstrated that disease-related symptoms returned to baseline levels within 1 week of discontinuing ruxolitinib. After 3 years of follow-up, no consistent pattern of AEs has been observed that would suggest a severe inflammatory syndrome after ruxolitinib discontinuation from either of the COMFORT trials. Nonetheless it is important to be aware that spleen size and symptoms will revert to baseline (or worse if the patient has progressed during ruxolitinib therapy), therefore careful dose tapering or concomitant steroids are considered when discontinuing ruxolitinib. The fact that two JAK inhibitors have been withdrawn due to neurological toxicity, one specifically described as Wernicke's encephalopathy, is indicative of the need to have heightened vigilance for these toxicities in patients being treated with novel agents. It will be important to understand whether there is an effect of JAK/STAT inhibition upon thiamine metabolism or critical neurological pathways which rely upon JAK/STAT. Lastly, increased risk of infection was noted in ruxolitinib-treated patients from the COMFORT studies. A number of case reports have also highlighted a number of severe infectious complications arising in ruxolitinib treated patients, including individual reports of cryptococcus neoformans pneumonia, bilateral toxoplasma retinitis, hepatitis $\mathrm{B}$ and tuberculosis reactivation ${ }^{26-28}$ as well as one case of progressive multifocal leucoencephalopathy, ${ }^{29}$ suggesting that ruxolitinib may have immunosuppressive effects which have implications in patient management.

In addition to JAK/STAT, other related pathways have been found to be dysregulated in MF and are beginning to be exploited therapeutically. For example, abnormalities in histone acetylation, PI3K/AKT, MEK and hedgehog signalling as well as telomerase TGF- $\beta 1$, mTOR and p53 were observed; each of these are potential therapeutic targets. Improved efficacy of combinations of JAK2 and either hedgehog, HDAC and PI3K inhibitors were also recently reported, albeit in mouse models, and a number of clinical trials are currently underway. Logically, interest is growing in our ability to improve patient outcomes using such combinations of JAK inhibitors and traditional and/or experimental agents. Combination studies offer patients the opportunity to move towards ultimately attaining a possible cure or at least deeper/more meaningful responses. However, challenges lie ahead in understanding how to assess the benefits of such approaches, since observation of a survival benefit or leukaemia-free survival would require very large trials conducted over a long period. Spleen volume response has almost become the standard primary endpoint in MF, but other measures may be more appropriate. Further efforts may help us to identify surrogate markers of response that could enable more rapid advancement in the selection of novel therapies or combinations, for example allele burden, CD34+, fibrosis grade, and/or other genetic signatures.

\section{Challenges ahead in the management of primary myelofibrosis, essential thrombocythaemia and polycythaemia vera}

It is very encouraging to witness the recent developments in the understanding and treatment of MPN and observe the benefits that these new options can provide to patients. In terms of translational research, if the events surrounding either progression to MF or indeed acute leukaemia were better understood, more biologically significant targets may be identified. Equally, a greater understanding of biological aspects of the disease will hopefully lead to better defined surrogate markers for highlighting both the patients in need of novel therapies and (once they are on these agents) the patients that are truly responding. It will also be important to assess the long-term safety and efficacy of new treatments, such as JAK inhibitors. Lastly, the introduction of these and other novel therapies is highlighting the need to determine both the current prevalence of disease and the true societal cost of both the disease and its treatments. This is poorly understood for MPN and so efforts should also focus on this area.

\section{References}

1 James C, Ugo V, Le Couedic JP et al. A unique clonal JAK2 mutation leading to constitutive signalling causes polycythaemia vera. Nature 2005;434:1144-8.

2 Dawson MA, Bannister AJ, Gottgens B et al. JAK2 phosphorylates histone H3Y41 and excludes HP1alpha from chromatin. Nature 2009;461:819-22.

3 Scott LM, Tong W, Levine RL et al. JAK2 exon 12 mutations in polycythemia vera and idiopathic erythrocytosis. $N$ Engl J Med 2007;356:459-68.

4 Pikman Y, Lee BH, Mercher T et al. MPLW515L is a novel somatic activating mutation in myelofibrosis with myeloid metaplasia. PLoS Med 2006;3:e270.

5 Klampfl T, Gisslinger H, Harutyunyan AS et al. Somatic mutations of calreticulin in myeloproliferative neoplasms. $N$ Engl J Med 2013;369:2379-90.

6 Nangalia J, Massie CE, Baxter EJ et al. Somatic CALR mutations in myeloproliferative neoplasms with nonmutated JAK2. $N$ Engl J Med 2013;369:2391-405.

7 Vannucchi AM, Lasho TL, Guglielmelli P et al. Mutations and prognosis in primary myelofibrosis. Leukemia 2013;27:1861-9.

8 Landolfi R, Marchioli R, Kutti J et al. Efficacy and safety of lowdose aspirin in polycythemia vera. N Engl J Med 2004;350:114-24.

9 Harrison C, Barbui T. Aspirin in low-risk essential thrombocythemia, not so simple after all? Leuk Res 2011;35:286-9.

10 Marchioli R, Finazzi G, Specchia G et al. Cardiovascular events and intensity of treatment in polycythemia vera. $N$ Engl J Med 2013;368:22-33.

11 Campbell PJ, MacLean C, Beer PA et al. Correlation of blood counts with vascular complications in essential thrombocythemia: analysis of the prospective PT1 cohort. Blood 2012;120:1409-11. 
12 Wilkins BS, Erber WN, Bareford D et al. Bone marrow pathology in essential thrombocythemia: interobserver reliability and utility for identifying disease subtypes. Blood 2008;111:60-70.

13 Passamonti F, Rumi E, Pietra D et al. A prospective study of 338 patients with polycythemia vera: the impact of JAK2 (V617F) allele burden and leukocytosis on fibrotic or leukemic disease transformation and vascular complications. Leukemia 2010;24:1574-9.

14 Gowin K, Thapaliya P, Samuelson J et al. Experience with pegylated interferon alpha-2a in advanced myeloproliferative neoplasms in an international cohort of 118 patients. Haematologica 2012;97:1570-3.

15 Kiladjian JJ, Cassinat B, Chevret S et al. Pegylated interferon-alfa-2a induces complete hematologic and molecular responses with low toxicity in polycythemia vera. Blood 2008;112:3065-72.

16 Kiladjian JJ, Masse A, Cassinat B et al. Clonal analysis of erythroid progenitors suggests that pegylated interferon alpha-2a treatment targets JAK2V617F clones without affecting TET2 mutant cells. Leukemia 2010;24:1519-23.

17 Verstovsek S, Passamonti F, Rambaldi A et al. Durable responses with the JAK1/ JAK2 inhibitor, INCB018424, in patients with polycythemia vera (PV) and essential thrombocythemia (ET) refractory or intolerant to hydroxyurea (HU). Blood (ASH Annu Meet Abs) 2010;116:313

18 Andersen CL, McMullin MF, Ejerblad E et al. A phase II study of vorinostat (MK-0683) in patients with polycythaemia vera and essential thrombocythaemia. Br J Haematol 2013;162:498-508.

19 Finazzi G, Vannucchi AM, Martinelli V et al. A phase II study of Givinostat in combination with hydroxycarbamide in patients with polycythaemia vera unresponsive to hydroxycarbamide monotherapy. Br J Haematol 2013;161:688-94.

20 Baerlocher GM, Leibundgut EO, Ayran C et al. Imetelstat rapidly induces and maintains substantial hematologic and molecular responses in patients with essential thrombocythemia (ET) who are refractory or intolerant to prior therapy: Preliminary phase II results. Blood (ASH Annu Meet Abs) 2012;120:179.
21 Reilly JT, McMullin MF et al. Guideline for the diagnosis and management of myelofibrosis. Br J Haematol 2012;158:453-71.

22 Verstovsek S, Gotlib J, Levy RS et al. Long-term outcomes of ruxolitinib therapy in patients with myelofibrosis: 3-Year update from COMFORT-I. Blood (ASH Annu Meet Abs) 2013;122:396.

23 Cervantes F, Vannucchi AM, Kiladjian JJ et al. Three-year efficacy, safety, and survival findings from COMFORT-II, a phase 3 study comparing ruxolitinib with best available therapy for myelofibrosis. Blood 2013;122:4047-53.

24 Keohane C, Radia DH, Harrison CN. Treatment and management of myelofibrosis in the era of JAK inhibitors. Biologics 2013;7:18998.

25 Tefferi A, Pardanani A. Serious adverse events during ruxolitinib treatment discontinuation in patients with myelofibrosis. Mayo Clin Proc 2011;86:1188-91.

26 Caocci G, Murgia F, Podda L et al. Reactivation of hepatitis B virus infection following ruxolitinib treatment in a patient with myelofibrosis. Leukemia 2014;28:225-7.

27 Wysham NG, Sullivan DR, Allada G. An opportunistic infection associated with ruxolitinib, a novel janus kinase 1,2 inhibitor. Chest 2013;143:1478-9.

28 Goldberg RA, Reichel E, Oshry LJ. Bilateral toxoplasmosis retinitis associated with ruxolitinib. N Engl J Med 2013;369:681-3.

29 Wathes R, Moule S, Milojkovic D. Progressive multifocal leukoencephalopathy associated with ruxolitinib. $N$ Engl J Med 2013;369:197-8.

Address for correspondence: Prof CN Harrison, Guy's and St Thomas' NHS Foundation Trust, Guy's Hospital, Great Maze Pond London, SE1 9RT, UK.

Email: claire.harrison@gstt.nhs.uk 